\title{
CORRELATIONS BETWEEN THE THERMOELECTRIC POWER AND HALL EFFECT OF SN- OR GE-DOPED IN $\mathrm{IN}_{2} \mathrm{O}_{3}$ POLYCRYSTALLINE CERAMICS
}

\author{
C. MARCEL* AND J. SALARDENNE \\ Laboratoire d'Etudes pour la Microélectronique, Université de Bordeaux I, 351 Cours de la Libération, \\ 33405 Talence cédex, FRANCE
}

\author{
S. Y. HUANG AND G. CAMPET AND J. PORTIER \\ Laboratoire de Chimie du Solide du CNRS, Université de Bordeaux I, 351 Cours de la Libération, 33405 \\ Talence cédex, FRANCE
}

(Received January 3 1996; In final form March 29 1996)

The thermoelectric power and Hall effect of Sn-or Ge-doped $\operatorname{In}_{2} \mathrm{O}_{3}$ ceramics are investigated based on a comparative study. The metal-type conductivity in both the samples occurs when the carrier concentration exceeds $\sim 10^{19} \mathrm{~cm}^{-3}$. The carrier mobility is found to be higher for Ge-doped samples. The relation between the $<<$ Lewis acid strength $>>$ of the dopant element and its scattering cross section is also presented.

\section{INTRODUCTION}

The simultaneous occurrence of high optical transparency (more than $80 \%$ ) in the visible region and high electrical conductivity $\left(\sigma>10^{3} \Omega^{-1} \cdot \mathrm{cm}^{-1}\right)$ is an unusual phenomenum found in a few non-stoichiometric oxides. The conditions to be met in such an oxide would appear to be following:

(i) The stoichiometric parent compound should be an insulator with a conduction-band edge $\mathrm{E}_{\mathrm{c}}$ lying at least $2.5 \mathrm{eV}$ above the top of the valence band and at least $2.5 \mathrm{eV}$ below the next higher conduction band. This condition is fulfilled in indium and tin oxides where the conduction band is the broad metal-5s band, the next higher band is the metal-5p band, and the valence band is the $\mathrm{O}^{2-}: 2 \mathrm{p}^{6}$ band.

(ii) Doping the parent compound n-type must result in a degenerate conduction band with a low enough carrier concentration so that the plasma frequency $\omega_{\mathrm{p}}$ associated with the free carriers remains below the visible range and no electrons are trapped in localizated states below the conduction-band edge. It is the latter part of this second condition that appears to be difficult to fulfill in transition-metal compounds.

*Corresponding author. 
In the case of indium-oxide films, doping the parent compound has been accomplished by introducing oxygen vacancies, $\operatorname{In}_{2} \mathrm{O}_{3-\mathrm{y}}(\mathrm{IO})$, and also by substituting quadrivalent $\mathrm{Sn}^{4+}$ for $\mathrm{In}^{3+}$ ions, $\mathrm{In}_{2-\mathrm{x}} \mathrm{Sn}_{\mathrm{x}} \mathrm{O}_{3-\mathrm{y}}$ (ITO) [1,2]. In ITO films, the oxygen-vacancy concentration can be suppressed $(y<x)$ and segregation of $\mathrm{SnO}_{2-\epsilon}$ as a second phase limits the concentration $\mathrm{n}$ of mobile charge carriers. A $\mathrm{y} \ll<\mathrm{x}$ makes the electrical conductivity of ITO films, which can be as high as $10^{4}$ $\Omega^{-1} . \mathrm{cm}^{-1}$, primarily mobility-limited by scattering from the donor dopant ions $[1,5]$.

In spite of the high conductivity $\sigma$ obtained in the best ITO films, modern applications demand higher values.

Given an upper limit on the charge carrier concentration ' $n$ ', which appears to be imposed by an intrinsic reduction limit of the In-O array [4,5], the search for a higher $\sigma=$ ne $\mu$ must turn to search for a higher electronic mobility $\mu$. That has to occur through the use of more appropriate dopants than Sn. In fact, we have recently shown $[4,6,7]$ that the ideal doping cation must have a low electronegativity and a small ionic radius $(r)$ associated with high effective nuclear charge $\left(Z^{*}\right)$. Indeed, such a cation having high value of $Z^{*} / r^{2}$ will polarize the electron cloud of oxygen $2 \mathrm{p}^{6}$ valence band more strongly, thereby screening its charge so as to weaken it as a scattering center. Moreover, a low electronegativity for the dopant cation accounts for a weak interaction between the conduction band electrons and the dopant cation. Zhang [8] established an empirical equation relating the Lewis acid strength of the cation, $\mathrm{L}$, its electronegativity, and the $\mathrm{Z} * / \mathrm{r}^{2}$ value as

$\mathrm{L}=\mathrm{Z} * \mathrm{r}^{2}-7.7_{\mathrm{x}}+8.0$

Under such a circumstance, a high $L$ value of the doping cation necessarily means a reduced scattering effect (and thereby a reduced scattering cross section) of the doping cation with regard to the conduction band electrons. Therefore, when the factor dominating the mobility is the scattering of electrons from the ionized donor centers, higher (lower) mobilities will occur for semiconductors doped with donor elements having higher (lower) $\mathrm{L}$ values [4,6,7]. Following this guideline, it appeared that the use of $\mathrm{Ge}^{4+}$ as a doping element in ITO (partially or totally substituted to $\mathrm{Sn}^{4+}$ ) could induce an enhancement in the mobility since [8]

$\mathrm{L}_{\mathrm{Ge}^{4+}}=3.06>\mathrm{L}_{\mathrm{Sn}^{4+}}=1.62$

In the present study, our main emphasis will be on complementing the choice of germanium as a dopant using both thermoelectric power and Hall effect measurements performed on a series of $\operatorname{In}_{2} \mathrm{O}_{3}$ samples either undoped or doped with $\mathrm{Sn}$ or Ge. The experiments have been carried out on single crystals and ceramics.

\section{PRELIMINARY CONSIDERATIONS RELATED TO THE THERMOELECTRIC POWER}

In this section, we shall recapitulate some of the important relations that will be used later in this article. The thermoelectric power $(\alpha)$, which is the temperature gradient of the total e.m.f, is generally defined as $[10,14]$ 
$\alpha=\Delta \mathrm{E} / \Delta \mathrm{T}=\Delta\left(\phi-\mu^{*} \mathrm{kT} / \mathrm{q}\right) / \Delta \mathrm{T}$

where, $\phi$ and $\mu^{*}$ designate the electrostatic and reduced electrochemical potentials respectively. In the case of completely non-degenerate semiconductors, Fistul established the following relations [11]

$\alpha_{1}=-\mathrm{k} / \mathrm{q}\left(2-\mu^{*}\right)$

$\alpha_{\mathrm{i}}=-\mathrm{k} / \mathrm{q}\left(4-\mu^{*}\right)$

where $\alpha_{1}$ and $\alpha_{i}$ symbolize the thermoelectric powers in a particular case of the carriers whose scattering is mainly limited by phonons and by ions respectively, and ' $k$ ' is the Boltzmann constant. Let us recall that for totally non-degenerate semiconductors, the value of the reduced potential is less than -2 , a value lower than that for the degeneracy limit, $\mu^{*}=-2$. Under such a circumstance, the dependence of $\alpha$, as a function of the conduction band carrier concentration, ' $n$ ', can be evaluated. Indeed, whatever scattering mechanism intervenes (phonon scattering or impurity scattering) ' $n$ ' sensibly varies as [16]

$\mathrm{n}=\left(\pi \mathrm{N}_{\mathrm{c}} / 2\right) \cdot \mathrm{e}^{\mu_{*}}$

$\mathrm{Nc}=4 \pi\left(2 \mathrm{~m} * \mathrm{kT} / \mathrm{h}^{2}\right)^{3 / 2}$ represents the effective density of states in the conduction band. Therefore, expressions (4) and (5) become, respectively

$\alpha_{1}=-\mathrm{k} / \mathrm{q}\left[2-\ln \left(2 \mathrm{n} / \pi \mathrm{N}_{\mathrm{c}}\right)\right]$

$\alpha_{\mathrm{i}}=-\mathrm{k} / \mathrm{q}\left[4-\ln \left(2 \mathrm{n} / \pi \mathrm{N}_{\mathrm{c}}\right)\right]$

On the other hand, in the degenerate semiconductor case $\left(\mu^{*}>>0\right)$, Fistul established the formulae [15]

$\alpha_{1}=-\mathrm{k} / \mathrm{q} \cdot \pi^{2} / 3 \cdot 1 / \mu^{*}$

$\alpha_{\mathrm{i}}=-\mathrm{k} / \mathrm{q}\left(\pi^{2} \mu^{*}\right) /\left(\pi^{2}+\mu^{*} 2\right) \sim-\mathrm{k} / \mathrm{q} \cdot \pi^{2} / \mu^{*}$

In the case of carrier scattering by phonons or by impurity ions, the conduction band carrier concentrations vary respectively as $[13,16]$

$\mathrm{n}_{1} \sim \mathrm{N}_{\mathrm{c}} \cdot \mu^{* 3 / 2}$

$\mathrm{n}_{\mathrm{i}} \sim \mathrm{N}_{\mathrm{c}} \cdot \mu^{* 3 / 2}$

Substituting the equations (11) and (12), respectively, in (9) and (10), we observe that $\alpha_{1}$ and $\alpha_{i}$ vary as $n_{1}^{-2 / 3}$ and $n_{i}^{-2 / 3}$, respectively. 


\section{EXPERIMENTAL}

Either undoped IO ceramics or IO ceramics doped with $\mathrm{Sn}$ or Ge, symbolized respectively as ITO and IGO, have been obtained. The undoped ceramics were prepared by heating the pellets $(10 \mathrm{~mm}$ diameter, $1 \mathrm{~mm}$ thick $)$ to various temperatures $\left(1100^{\circ} \mathrm{C}, 1200^{\circ} \mathrm{C}, 1300^{\circ} \mathrm{C}\right.$ and $\left.1350^{\circ} \mathrm{C}\right)$ employing a heating rate of $100^{\circ} \mathrm{C} / \mathrm{h}$. The pellets were sintered at those temperatures for $24 \mathrm{~h}$. The ITO and IGO ceramics were prepared by intimately mixing the starting material oxides (i.e., $\mathrm{In}_{2} \mathrm{O}_{3}+\mathrm{SnO}_{2}$ or $\mathrm{GeO}_{2}$ ). The mixed powders were cold pressed in a steel die and then submitted to an isostatic pressure of 5 bars. The pellets so obtained were sintered in air for $24 \mathrm{~h}$. The heating and cooling rate $\left(100^{\circ} \mathrm{C} / \mathrm{hour}\right)$ was controlled by an Eurotherm programmer. All electrical measurements were performed using equipments described elsewhere [5,9].

\section{RESULTS AND DISCUSSION}

Values of the room temperature conductivity, carrier concentration, and mobility, all measured by Hall effect, for undoped and for $\mathrm{Sn}$ - or Ge-doped $\operatorname{In}_{2} \mathrm{O}_{3}$ ceramics are given in Table $I$. The increase (decrease) of $n(\mu)$, observed in samples a-c, obviously accounts for an enhancement in the oxygen-vacancy concentration, as mentioned above for undoped single crystals. As the dopant concentration is increased, ' $\mathrm{n}$ ' increases in the beginning for both Sn-doped (d-g) and Ge-doped (i-l) samples and later reaches a plateau (samples $\mathrm{g}, \mathrm{h}$ and $\mathrm{l}, \mathrm{m}$ ), correlated to the

TABLE I

Values of the carrier mobility, carrier concentration and room temperature conductivity for the undoped (samples a-c) and Sn- or Ge-doped $\mathrm{In}_{2} \mathrm{O}_{3}$ (samples d-m) ceramics [3].

\begin{tabular}{|c|c|c|c|c|}
\hline $\begin{array}{l}\mathrm{M} /(\mathrm{In}+\mathrm{M}) \\
\mathrm{M}=\mathrm{Sn} \text { or } \mathrm{Ge} \text { (\% values) }\end{array}$ & Sample symbol & $\mu_{H}\left(\mathrm{~cm}^{2} \mathrm{v}^{-1} \mathrm{~s}^{-1}\right)$ & $\mathrm{n}_{\mathrm{H}} \cdot 10^{18}\left(\mathrm{~cm}^{-3}\right)$ & $\mathrm{s}_{\mathrm{H}} \cdot 10(\mathrm{~S} / \mathrm{cm})$ \\
\hline $\begin{array}{l}\mathrm{Sn} /(\mathrm{In}+\mathrm{Sn}) \\
\text { sintered at } 1100^{\circ} \mathrm{C}\end{array}$ & $\mathbf{a}$ & 40 & 0.13 & 0.083 \\
\hline $\begin{array}{l}\mathrm{Sn} /(\text { In+Sn) } \\
\text { sintered at } 1200^{\circ} \mathrm{C}\end{array}$ & b & 45 & 0.15 & 0.108 \\
\hline $\begin{array}{l}\mathrm{Sn} /(\mathrm{In}+\mathrm{Sn}) \\
\text { sintered at } 1350^{\circ} \mathrm{C}^{* *}\end{array}$ & c & 25 & 0.40 & 0.160 \\
\hline $\mathrm{Sn} /(\mathrm{In}+\mathrm{Sn})=0.125$ & d & 35.1 & 40.0 & 25.30 \\
\hline $\mathrm{Sn} / /_{(\mathrm{In}+\mathrm{Sn})}=0.250$ & $\mathrm{e}$ & 30.9 & 100 & 49.40 \\
\hline $\mathrm{Sn} / /_{(\mathrm{In}+\mathrm{Sn})}=0.500$ & f & 29.0 & 180 & 86.30 \\
\hline $\mathrm{Sn} / /_{(\mathrm{In}+\mathrm{Sn})}=1.300$ & g & 28.0 & 190 & 85.10 \\
\hline $\mathrm{Sn} / /_{(\mathrm{In}+\mathrm{Sn})}=2.500$ & $\mathrm{~h}$ & 25.0 & 190 & 76.40 \\
\hline $\mathrm{Sn} / /_{(\mathrm{In}+\mathrm{Ge})}=0.125$ & $\mathrm{i}$ & 45.0 & 32.0 & 23.00 \\
\hline $\mathrm{Sn} /_{(\mathrm{In}+\mathrm{Ge})}=0.250$ & $\mathrm{j}$ & 50.0 & 80.0 & 64.00 \\
\hline $\mathrm{Sn} /_{(\mathrm{In}+\mathrm{Ge})}=0.500$ & $\mathrm{k}$ & 55.0 & 180 & 158.0 \\
\hline $\mathrm{Sn} / /_{(\mathrm{In}+\mathrm{Ge})}=1.300$ & 1 & 52.0 & 190 & 156.0 \\
\hline $\mathrm{Sn} / /_{(\mathrm{In}+\mathrm{Ge})}=2.500$ & m & 50.0 & 190 & 152.0 \\
\hline
\end{tabular}

*The ratio $\mathrm{M} /(\mathrm{In}+\mathrm{M})$ corresponds to $10^{2} \mathrm{x}$ in $\mathrm{In}_{2-2 \mathrm{x}} \mathrm{Sn}_{2 \mathrm{x}} \mathrm{O}_{3}$ or in $\mathrm{In}_{2-2 \mathrm{x}} \mathrm{Ge}_{2 \mathrm{x}} \mathrm{O}_{3}$.

**No noticeable change in conductivity occurs when the sintering temperature is increased above $1350^{\circ} \mathrm{C}$ [7], so all the $\mathrm{Sn}$ - or Ge-doped samples have been sintered at $1350^{\circ} \mathrm{C}$. 
formation of a second phase $\left(\mathrm{SnO}_{2-\varepsilon}\right.$ or $\left.\mathrm{GeO}_{2-\varepsilon}\right)$ as evidenced by $\mathrm{X}$-ray analysis. Moreover, the grain boundary effect inhibits the normally expected increase in the carrier mobility with the Sn ratio (samples $d-h$ ), which has been observed for single crystals (see Table I, samples a-c). However, the grain boundary effects do not entirely hinder the <<beneficial>> effect of Ge-doping on $\mu$. As it is clear from Table I despite being ceramics, Ge-doped samples show an increase in $\mu$, which has been observed for Sn-doped samples. It is important to note that, for similar carrier concentrations, $\mu$ is about $50 \%$ (or more) higher for IGO than for ITO.

The thermoelectric power, like the mobility, can be governed by the scattering mechanisms of the carriers. Therefore, we can expect different values of $\alpha$ for $\operatorname{In}_{2} \mathrm{O}_{3}$ heavily doped with $\mathrm{Sn}$ or Ge impureties. That is illustrated hereafter for samples heavily doped with $\mathrm{Sn}(\mathrm{e}-\mathrm{h})$ or $\mathrm{Ge}(\mathrm{j}-\mathrm{m})$. It is clear from Fig. 1 that $\alpha$ values, referring to samples $j-m$, are somewhat higher than those referring to samples $e-h$. This can be understood, using the equation (10), for the sake of simplicity, which is valid in the degenerate case. Using the expression (10) the basic nature can indeed be foreseen; one can write

$\alpha_{\mathrm{IGO}} / \alpha_{\mathrm{ITO}} \sim \mu^{*}{ }_{\mathrm{ITO}} / \mu^{*}{ }_{\mathrm{IGO}}$

And also $\mu^{*}$ depends upon the carrier mobility, the carrier effective mass and the scattering cross section $Q$ according to [12]

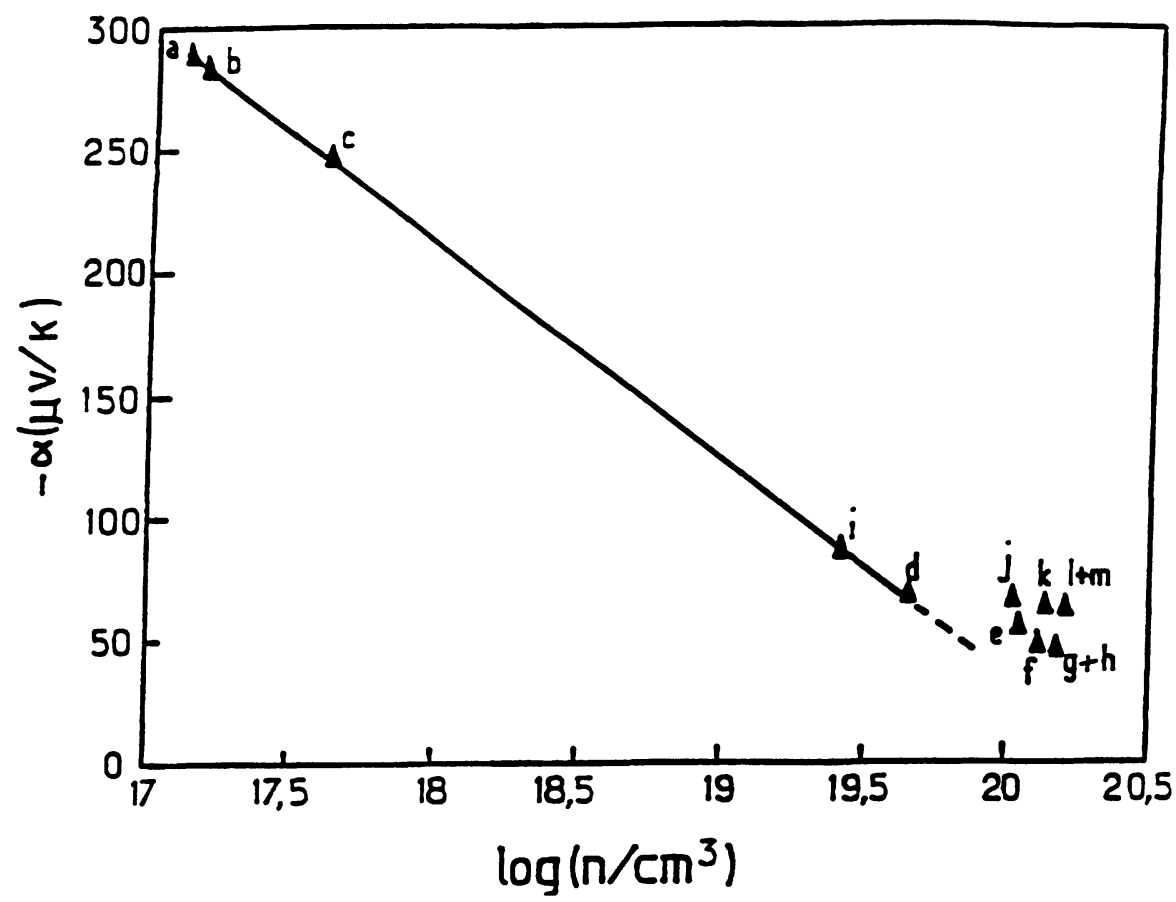

FIGURE 1 Dependence of Thermoelectric power on the electron density in undoped or Sn- or Ge-doped $\mathrm{In}_{2} \mathrm{O}_{3}$ ceramics at $300 \mathrm{~K}$. Numbers and symbols correspond to the same samples as in Table I. 
$\mu^{*}=\mathrm{q}^{2} / 2 \mathrm{~m}^{*} \mathrm{n}^{2} \mu^{2} \mathrm{Q}^{2}$

Consequently, we can obtain the following expression after simple transformation

$\alpha_{\mathrm{IGO}} / \alpha_{\mathrm{ITO}}=\left[\mu_{\mathrm{IGO}} / \mu_{\mathrm{ITO}}\right]^{2}\left[\mathrm{Q}_{\mathrm{Ge}^{4+}} / \mathrm{Q}_{\mathrm{Sn}^{4-}}\right]^{2}$

Using the relation (14), the $\mu$ and $\alpha$ values, given respectively in Table I and in Fig. 1 , one gets

$\mathrm{Q}_{\mathrm{Ge}^{4+}} / \mathrm{Q}_{\mathrm{Sn}^{4+}} \sim 0.55$

It is interesting to note that for similarly heavily doped ITO and IGO (such as f and k) the value obtained above is close to the ratio calculated based on the Lewis acid strengths [8]. Using relation (2) we get

$\mathrm{L}_{\mathrm{Sn}^{4+}} / \mathrm{L}_{\mathrm{Ge}^{4+}} \sim 0.53$

This result confirms the expected close relation between the scattering cross section of the dopant ion and its Lewis acid strength. It appears that when the factor dominating the mobility is the scattering of electrons from the ionized donor centers, $L$ roughly varies inversely as $Q$.

We note here that the concepts we have put forward also apply for other degenerate oxides having a predominant ionic-bond character as we have recently investigated [6]. A priori, they may also be conveniently used for semiconductors having covalent-bond character such as $\mathrm{Si}$ or Ge heavily doped by As or P ( $\mathrm{n}>>$ $10^{19} \mathrm{~cm}^{-3}$ as it occured here for ITO and IGO) [12]. In fact, the $<<$ Lewis acid strength $>>$ of phosphorous is larger than that of As which is in good agreement with expectation. One apparently has [12]

$\alpha_{\text {P doped Si }}>\alpha_{\text {As doped Si }}$

and

$\alpha_{P \text { doped Ge }}>\alpha_{\text {As doped Ge }}$

However, further investigation is necessary if one wishes to extend the simple concepts we have proposed here for ITO and IGO, to other semiconductors having, contrarily to ITO and IGO, a strong covalent-bond character.

\section{CONCLUSIONS}

An attempt has been made to compare the properties of $\mathrm{Sn}$-doped and Ge-doped $\mathrm{In}_{2} \mathrm{O}_{3}$ polycristalline ceramics. Thermoelectric power and Hall effect measurements have been carried out on all samples. It is evident that the Ge-doped samples 
possess higher mobility compared to the Sn-doped samples with the same carrier concentration. An empirical correlation of the Lewis acid strengths of the two ions to their scattering cross sections, which can be used as a mean to choose the doping cation, has also been suggested.

\section{REFERENCES}

1. K.L. Chopra, S. Major and D.K. Pandya, Thin Solid Films, 102, 1 (1983).

2. H.W. Lahman and R. Whider, Thin Solid Films, 27, 359 (1969).

3. I. Hamberg and C.G. Granqvist, J. Appl. Phys. 60, (1986), 123 and references therein.

4. S.J. Wen, G. Campet, J. Portier, G. Couturier and J.B. Goodenough, Mat. Sci. and Eng. B, 14, (1992), 115

5. S.J. Wen, G. Couturier, J.P. Chaminade, E. Marquestaut, J. Claverie and P. Hagenmüller, J. Solid State Chem. 101(2), (1993), 203.

6. G. Campet, S.D. Han, S.J. Wen, J.P. Manaud, J. Portier, Y. Xu and J. Salardenne, Mat. Sci. and Eng. B (accepted for publication (1995)).

7. S.J. Wen, doctoral thesis, University of Bordeaux I, (1992).

8. Y. Zhang, Inorganic Chem. 21, (1982), 3886.

9. P. Dordor, E. Marquestaut, Rev. Phys. Appl., 15, (1980), 1607.

10. V.I. Fistul, $<<$ Heavily doped Semiconductors $>>$ Plenum Press, New York, (1969).

11. Reference 10, p. 48.

12. Reference 12, pp 144 and 145.

13. Y. Marfaing, <<Interface Semiconductor Electrolyte>>, CNRS, Ecole d'Hiver, Aussois, 9-15, December, (1984), p. 10.

14. Reference $10, \mathrm{pp} \mathrm{47,} 48$ and 140.

15. Reference 10 p. 143.

16. Reference $10 \mathrm{pp} 53$ and 104-106. 

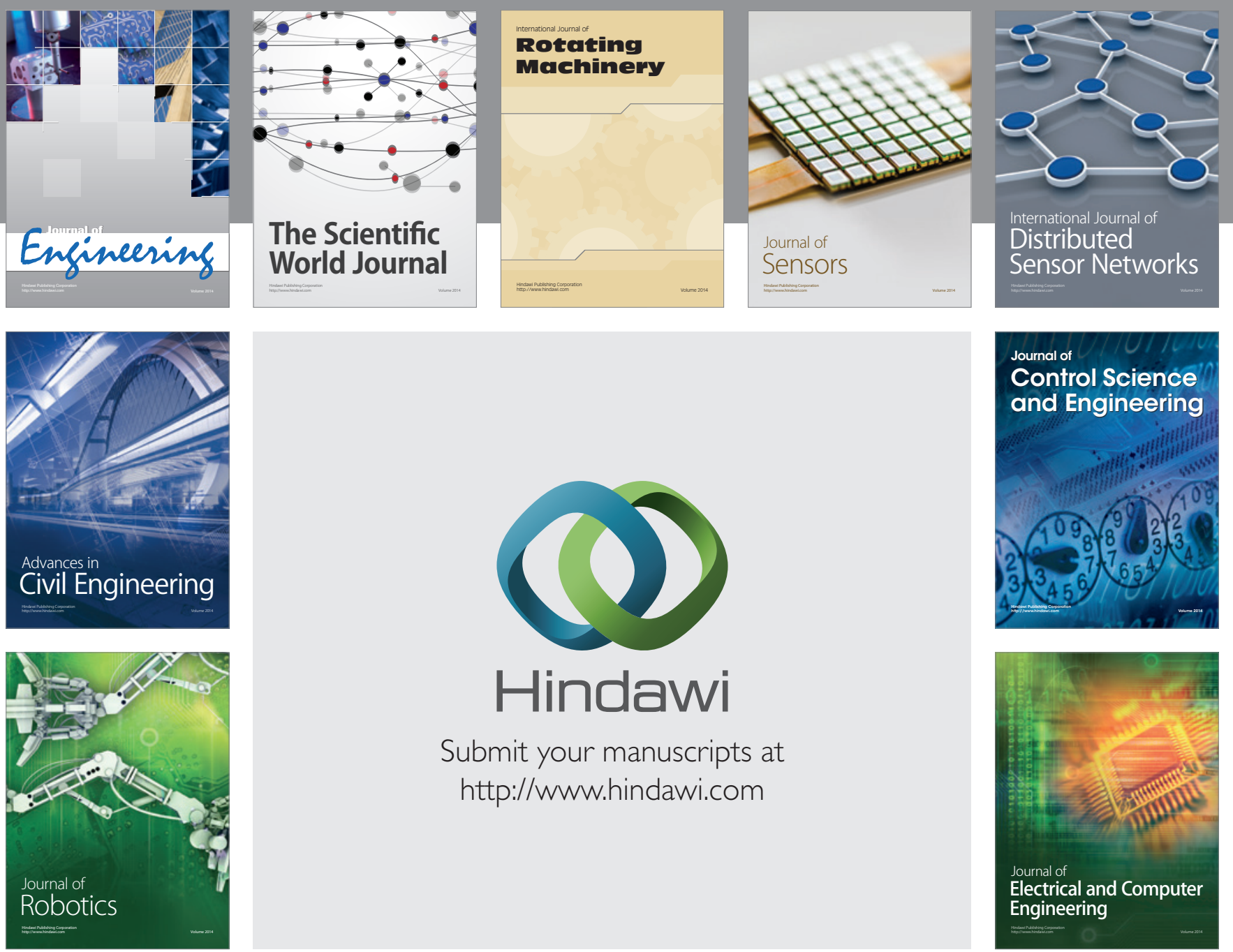

Submit your manuscripts at

http://www.hindawi.com
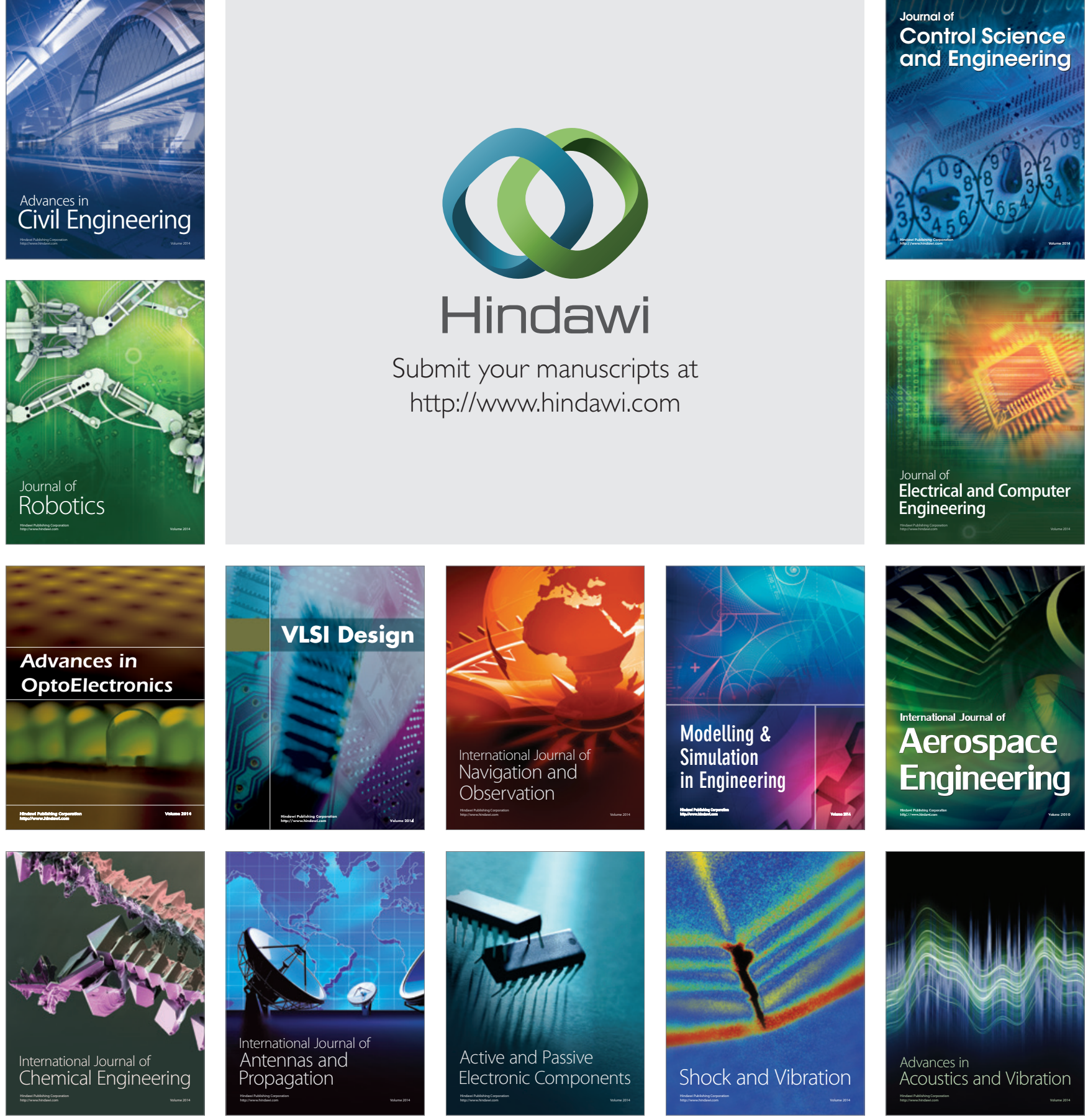